\title{
A NOTE ON LOGHARMONIC MAPPINGS
}

\section{ZAYID ABDULHADI}

Received 5 January 2002

We consider the problem of minimizing the moments of order $p$ for a subclass of logharmonic mappings.

2000 Mathematics Subject Classification: 30C55, 30C62, 49Q05.

1. Introduction. Let $H(U)$ be the linear space of all analytic functions defined on the unit disc $U=\{z=x+i y:|z|<1\}$. A logharmonic mapping is a solution of the nonlinear elliptic partial differential equation

$$
\overline{f_{\bar{z}}}=(a \bar{f} / f) f_{z},
$$

where the second delatation function $a$ is in $H(U)$ and $|a(z)|<1$ for all $z \in U$. If $f$ does not vanish on $U$, then $f$ is of the form

$$
f=H \cdot \bar{G},
$$

where $H$ and $G$ are in $H(U)$. On the other hand, if $f$ vanishes at 0 but has no other zeros in $U$, then $f$ admits the representation

$$
f(z)=z^{m}|z|^{2 \beta m} h(z) \overline{g(z)},
$$

where

(a) $m$ is nonnegative integer,

(b) $\beta=\overline{a(0)}(1+a(0)) /\left(1-|a(0)|^{2}\right)$ and therefore, $\mathfrak{R} \beta>-1 / 2$,

(c) $h$ and $g$ are analytic in $U, g(0)=1$, and $h(0) \neq 0$.

Univalent logharmonic mappings on the unit disc have been studied extensively. For details see $[1,2,3,4,5,6,7,8]$. Suppose that $f$ is a univalent logharmonic mapping defined on the unit disc $U$. Then, if $f(0)=0$, the function $F(\zeta)=\log \left(f\left(e^{\zeta}\right)\right)$ is univalent and harmonic on the half plane $\{\zeta: \operatorname{Re} \zeta<0\}$. For more details on univalent harmonic mappings defined in the unit disc $U$, see [9, 10, 11, 12].

In this note, we consider the problem of minimizing the moments of order $p$ over a subclass of the class logharmonic mappings defined over the unit disc $U$. It is interesting to note that the extremal functions are univalent starlike logharmonic mappings.

\section{Moments of order $p$}

THEOREM 2.1. Let $f=z h(z) \overline{g(z)}$ be logharmonic mapping defined on the unit disc $U$ such that $h(0)=g(0)=1$. Let $M_{p}(r, f)$ denote the moment of order $p, p \geq 0$. Then,

$$
M_{p}(r, f) \geq 2 \pi\left(\frac{r^{p+2}}{p+2}-\frac{r^{p+4}}{p+4}\right) .
$$


Equality holds if and only if

$$
f_{1}(z)=z \frac{(1+((p+2) /(p+4)) \bar{z})}{(1+((p+2) /(p+4)) z)}
$$

or one of its rotations $\bar{\eta} f_{1}(\eta z)$.

REMARK 2.2. If $p=0$ in Theorem 2.1, then we have the problem of minimizing the area. Moreover, if $p=2$, then we obtain the minimum of the moment of inertia.

Proof. Let $f=z h(z) \overline{g(z)}$ be logharmonic mapping defined on the unit disc $U$. Then, $f$ satisfies (1.1) for some $a \in H(U)$ such that $|a(z)|<1$ and $a(0)=0$. Hence, using Schwarz's lemma, we have

$$
\begin{aligned}
M_{p}(r, f) & \geq \int_{0}^{r} \int_{0}^{2 \pi}|f|^{p}\left(\left|f_{z}\right|^{2}-\left|f_{\bar{z}}\right|^{2}\right) \rho d \theta d \rho \\
& =\int_{0}^{r} \int_{0}^{2 \pi}|f|^{p}\left|f_{z}\right|^{2}\left(1-|a|^{2}\right) \rho d \theta d \rho \\
& \geq \int_{0}^{r} \rho\left(1-\rho^{2}\right) \int_{0}^{2 \pi}|f|^{p}\left|f_{z}\right|^{2} d \theta d \rho .
\end{aligned}
$$

Writing $(h \cdot g)^{p / 2} \cdot(z h)^{\prime} \cdot g=1+\sum_{k=1}^{\infty} c_{k} z^{k}$, we have

$$
\int_{0}^{2 \pi}|f|^{p}\left|f_{z}\right|^{2} d \theta=2 \pi \rho^{p}\left(1+\sum_{k=1}^{\infty}\left|c_{k}\right|^{2} \rho^{2 k}\right)
$$

and therefore,

$$
M_{p}(r, f) \geq 2 \pi \int_{0}^{2 \pi} \rho^{p}\left(1-\rho^{2}\right) d \rho=2 \pi\left(\frac{r^{p+2}}{p+2}-\frac{r^{p+4}}{p+4}\right) .
$$

Equality holds if and only if

$$
(h)^{p / 2} \cdot(g)^{(p+2) / 2} \equiv 1
$$

and $a(z)=\eta z,|\eta|=1$. This implies that

$$
(h)^{(p+2) / 2} \cdot(g)^{p / 2} g^{\prime}=\eta
$$

and then,

$$
z \cdot \frac{\partial(h \cdot g)^{(p+2) / 2}}{\partial z}=\frac{(p+2)\left(1-(h \cdot g)^{(p+2) / 2}+\eta z\right)}{2} .
$$

The solution of the differential equation

$$
z \cdot u(z)^{\prime}+\frac{(p+2) \cdot u(z)}{2}=\frac{(p+2)(1+\eta z)}{2} ; \quad u(0)=1
$$


is $u(z)=(h(z) g(z))^{(p+2) / 2}=1+((p+2) /(p+4)) \eta z$. Together with (2.6), we get

$$
\frac{g(z)^{\prime}}{g(z)}=\frac{\eta}{(1+((p+2) /(p+4)) \eta z)}
$$

and therefore,

$$
\begin{aligned}
g(z) & =\left(1+\frac{p+2}{p+4} \eta z\right)^{(p+4) /(p+2)}, \\
z h(z) & =\frac{z}{(1+((p+2) /(p+4)) \eta z)},
\end{aligned}
$$

which leads to the solution $\bar{\eta} f_{1}(\eta z)$. Since

$$
\phi(z)=\frac{z h(z)}{g(z)}=\frac{z}{(1+((p+2) /(p+4)) \eta z)^{(2 p+6) /(p+2)}}
$$

is a starlike univalent analytic, it follows from [4, Theorem 2.1] that $f_{1}$ is a starlike univalent logharmonic mapping.

\section{REFERENCES}

[1] Z. Abdulhadi, Close-to-starlike logharmonic mappings, Int. J. Math. Math. Sci. 19 (1996), no. 3, 563-574.

[2] _ Typically real logharmonic mappings, Int. J. Math. Math. Sci. 31 (2002), no. 1, 1-9.

[3] Z. Abdulhadi and D. Bshouty, Univalent functions in $H \cdot \bar{H}(D)$, Trans. Amer. Math. Soc. 305 (1988), no. 2, 841-849.

[4] Z. Abdulhadi and W. Hengartner, Spirallike logharmonic mappings, Complex Variables Theory Appl. 9 (1987), no. 2-3, 121-130.

[5] _ Univalent harmonic mappings on the left half-plane with periodic dilatations, Univalent Functions, Fractional Calculus, and Their Applications (Kōriyama, 1988), Ellis Horwood Series: Mathematics and Its Applications, Horwood, Chichester, 1989, pp. 13-28.

[6] Univalent logharmonic extensions onto the unit disk or onto an annulus, Current Topics in Analytic Function Theory, World Scientific Publishing, New Jersey, 1992, pp. 1-12.

[7] __ One pointed univalent logharmonic mappings, J. Math. Anal. Appl. 203 (1996), no. 2, 333-351.

[8] , Polynomials in H H, Complex Variables Theory Appl. 46 (2001), no. 2, 89-107.

[9] J. Clunie and T. Sheil-Small, Harmonic univalent functions, Ann. Acad. Sci. Fenn. Ser. A I Math. 9 (1984), 3-25.

[10] W. Hengartner and G. Schober, Harmonic mappings with given dilatation, J. London Math. Soc. (2) 33 (1986), no. 3, 473-483.

[11] - On the boundary behavior of orientation-preserving harmonic mappings, Complex Variables Theory Appl. 5 (1986), no. 2-4, 197-208.

[12]___ Univalent harmonic functions, Trans. Amer. Math. Soc. 299 (1987), no. 1, 1-31.

Zayid Abdulhadi: Department of Computer Science, Mathematics and Statistics, AMERICAN UNIVERSITY OF SHARJAH, P.O. BOX 26666, SHARJAH, UNITED ARAB EMIRATES

E-mail address: zahadi@aus.ac.ae 


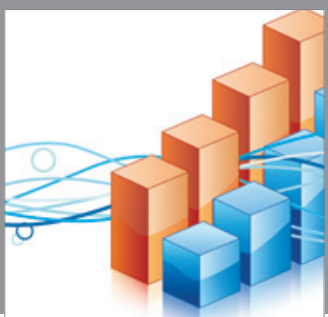

Advances in

Operations Research

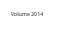

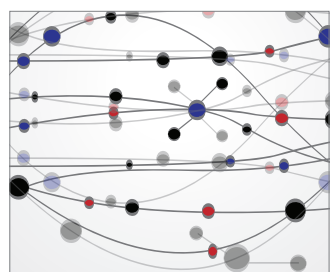

\section{The Scientific} World Journal
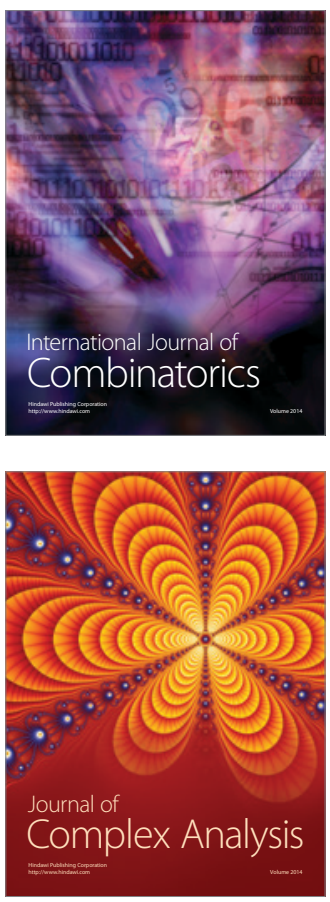

International Journal of

Mathematics and

Mathematical

Sciences
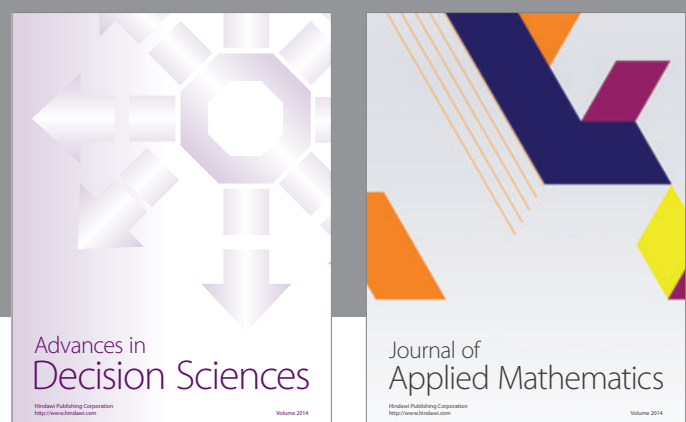

Journal of

Applied Mathematics
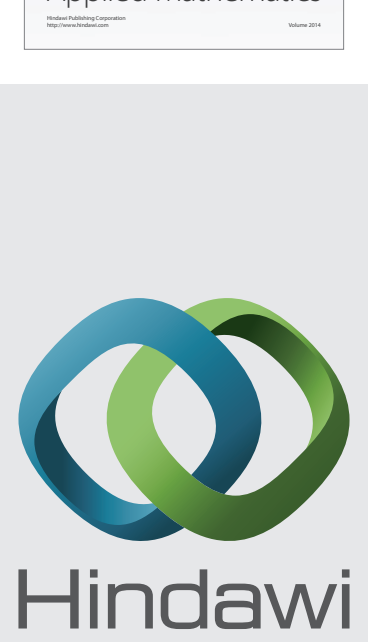

Submit your manuscripts at http://www.hindawi.com
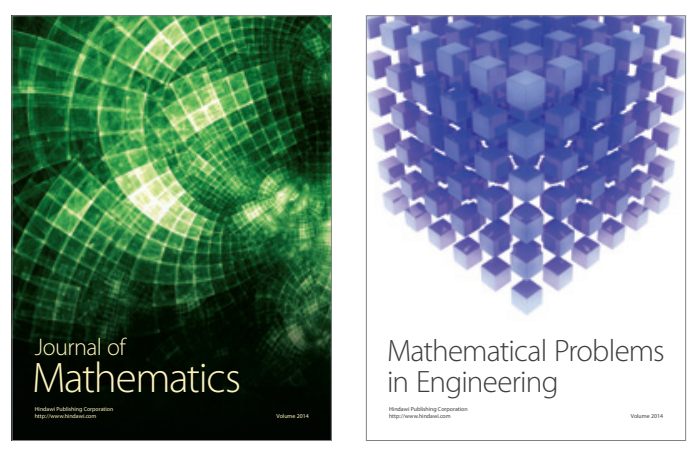

Mathematical Problems in Engineering
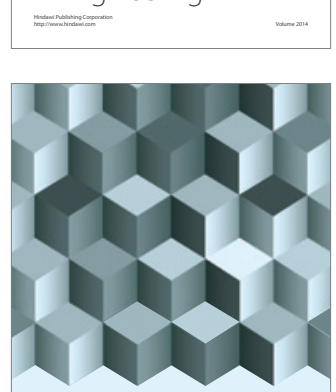

Journal of

Function Spaces
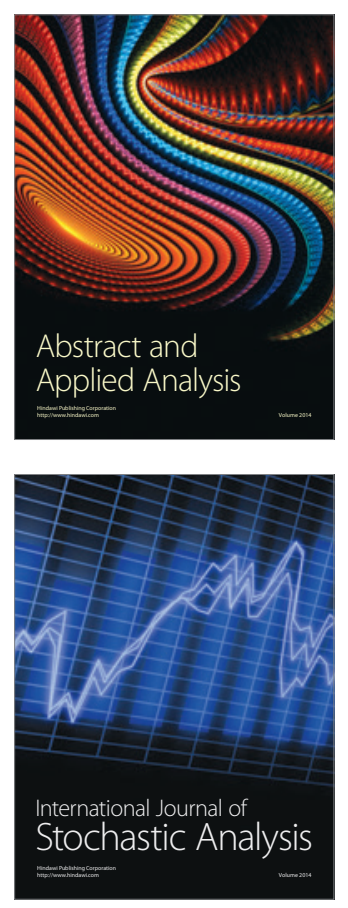

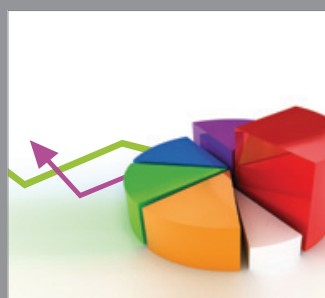

ournal of

Probability and Statistics

Promensencen
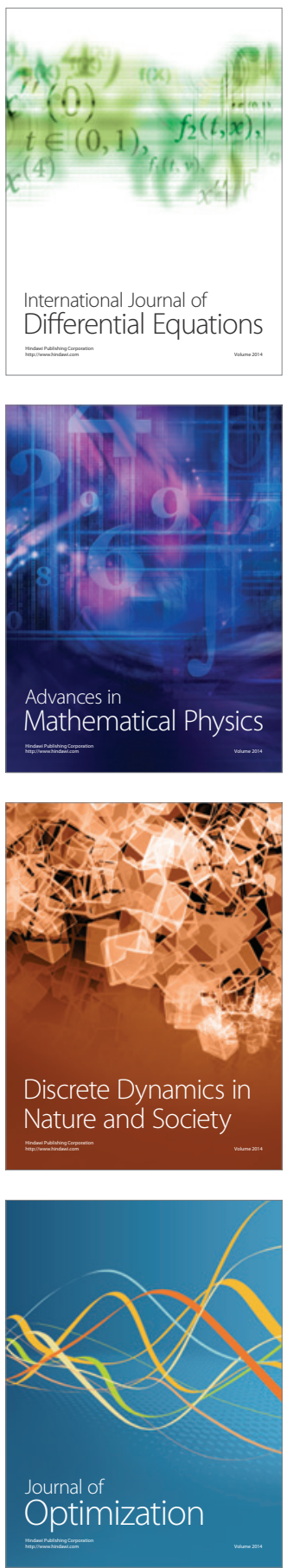